\title{
Effects of phorbol myristate acetate and sivelestat on the lung injury caused by fat embolism in isolated lungs
}

\author{
Chia-Chih Lin', Pei-Hsin Liu², Shang Jyh Kao ${ }^{3,4}$ and Hsing I Chen ${ }^{5^{*}}$
}

\begin{abstract}
Background: Fat embolism syndrome (FES) associated with acute lung injury (ALI) is a clinical condition following long bone fracture. We have reported 14 victims due to ALI with FES. Our laboratory has developed an animal model that produced fat emboli (FE). The major purpose of this study was to test whether neutrophil activation with phorbol myristate acetate (PMA) and inhibition with sivelestat (SVT) exert protection on the lung.

Methods: The lungs of Sprague-Dawley rats were isolated and perfused. FE was produced by addition of corn oil micelles into the lung perfusate. PMA and SVT were given simultaneously with FE. Parameters such as lung weight/body weight ratio, LW gain, exhaled nitric oxide (NO), protein concentration in bronchoalveolar lavage relating to ALI were measured. The neutrophil elastase (NE), myeloperoxidase, malondialdehyde and phopholipase $A_{2}$ activity were determined. We also measured the nitrate/nitrite, methyl guanidine (MG), and cytokines. Pulmonary arterial pressure and microvascular permeability were assessed. Lung pathology was examined and scored. The inducible and endothelial NO synthase (iNOS and eNOS) were detected.
\end{abstract}

Results: FE caused ALI and increased biochemical factors. The challenge also resulted in pulmonary hypertension and increased microvascular permeability. The NE appeared to be the first to reach its peak at $1 \mathrm{hr}$, followed by other factors. Coadministration with PMA exacerbated the FE-induced changes, while SVT attenuated the effects of FE.

Conclusions: The FE-induced lung changes were enhanced by PMA, while SVT had the opposite effect. Sivelestat, a neutrophil inhibitor may be a therapeutic choice for patients with acute respiratory distress syndrome (ARDS) following fat embolism.

Keywords: Fat embolism, Acute lung injury, Neutrophil elastase, Phorbol myristate acetate, Sivelestat

\section{Background}

Fat embolism syndrome (FES) is a serious clinical problem in patients associated with long bone fracture [1-3]. Although the precise mechanisms of FES remain unclear, intravasation of fat or fatty acids from broken long bones and other sources is the primary cause leading to FES $[3,4]$. In two clinical investigations, we have reported a total of 14 cases who died of acute respiratory distress syndrome (ARDS) associated with FES [1,3]. The occurrence of ARDS following FES suggests that the lung is one of the target organs following intravasation of fat emboli [1-4].

\footnotetext{
* Correspondence: chenhi@mail.tcu.edu.tw

${ }^{5}$ Institute of Physiological and Anatomical Medicine, Tzu Chi University, Hualien, Taiwan

Full list of author information is available at the end of the article
}

In order to elucidate the possible mediators involved in the ARDS associated with FES, we have developed an animal model that produces fat embolism in anesthetized rats. Intravenous administration of corn oil micelles induces alveolar edema and hemorrhage. The pathological changes are associated with fatty droplets and fibrin thrombi in the lung, kidney and brain. The arteriolar lumen is filled with fatty deposits. Hypoxia and hypercapnia ensue. Biochemical changes include increases in plasma phospholipase $\mathrm{A}_{2}$, nitrate/nitrite, methyl guanidine and proinflammatory cytokines [5]. The animal model has been used to study the protective effect of $\mathrm{N}$-Acetylcysteine [6], and the effects of inducible nitric oxide synthase (iNOS) inhibitors and nitric oxide donors 
[7]. Our results indicate that $\mathrm{N}$-acetylcysteine provides protection to the FES, while nitric oxide is detrimental.

Activation and recruitment of neutrophil that lead to the release of neutrophil elastase (NE) and other toxic mediators may play an initial role in the pathogenesis of ALI/ ARDS [8-10]. Accumulating evidence has indicated the involvement of neutrophil activation and NE induced by phorbol myristate acetate (PMA) and other agents [11-13]. Animal experimentation has demonstrated that sivelestat (SVT), an inhibitor of NE attenuates ALI via reduction of NE following lipopolysaccharide administration or inhalation $[14,15]$, and cardiopulmonary bypass $[16,17]$.

The present study was designed to test whether neutrophil activation with PMA and inhibition with SVT exert protective and/or detrimental effects on the acute lung injury caused by fat embolism.

\section{Materials and methods Animal preparation}

We used male Spague-Dawley (SD) rats, 12-14 wk-old, weighing 360-380 g. The animals were obtained from the National Animal Center and housed in the University Laboratory Animal Center with good environment control. The animal experiment was approved by the University Committee of Laboratory Animal Care and Use, and followed the guidelines of the National Animal Research Center. The room temperature was maintained at $21 \pm$ $1^{\circ} \mathrm{C}$ under a $12 / 12 \mathrm{hr}$ light/dark regimen. Food and water were provided ad libitum.

\section{Isolation and perfusion of the lung in situ}

We followed the procedures for the preparation of isolated and perfused rat's lungs in situ $[7,18]$. In brief, the rat's lungs were isolated and perfused with constant flow. Lung weight (LW) and LW gain (LWG) were recorded. Pulmonary arterial pressure (PAP) and pulmonary venous pressure (PVP) were measured.

\section{Microvascular permeability $\left(\mathrm{K}_{\mathrm{fc}}\right)$}

Capillary filtration coefficient $\left(\mathrm{K}_{\mathrm{fc}}\right)$ as an index of microvascular permeability was calculated from the increase in LW produced by an elevation in PVP. The $\mathrm{K}_{\mathrm{fc}}$ was defined as the initial weight gain rate $(\mathrm{g} / \mathrm{min})$ divided by PVP $\left(10 \mathrm{~cm} \mathrm{H}_{2} \mathrm{O}\right)$ and $\mathrm{LW}$, and expressed as $\mathrm{g} / \mathrm{min} /$ $\mathrm{cmH}_{2} \mathrm{O} / 100 \mathrm{~g}$. During the experiment, PVP was rapidly elevated by $10 \mathrm{~cm} \mathrm{H}_{2} \mathrm{O}$ for $7 \mathrm{~min}$ to measure $\mathrm{K}_{\mathrm{fc}}$. This hydrostatic challenge elicited a biphasic increase in LW: an initial rapid component, followed by slow and steady component. The slow component of the weight gain was plotted on a semilog scale as a function of time. The capillary filtration rate was obtained by extrapolating the slow component of the weight gain back to zero time $[7,18]$.

\section{Exhaled NO}

An increase in exhaled NO concentration has been used as an early marker of lung inflammation or injury $[19,20]$. We measured the NO concentration in expired air. A specimen of exhaled air (300 $\mathrm{ml}$ in $30 \mathrm{~min}$ ) was suctioned into a gas purge chamber previously evacuated to remove oxygen. The NO concentration was rapidly determined after air collection. The measurement of NO with a chemiluminescence analyzer (Sievers 270B NOA; Sievers Institute, Denver, CO, USA) was based on the principle that NO interacts with ozone to generate chemiluminescent light. The chemiluminescence is directly proportional to the NO level. In addition to a photomultiplier tube, an ozone generator and a gas chamber were included. The ozone generator was used to produce ozone internally. The exhaled NO was measured every 30 min after introduction of corn micelles into the lung perfusate. It reached its peak depending on the experimental conditions. The peak value was taken as the NO concentration.

\section{Protein concentration in bronchoalveolar lavage (PCBAL)}

After the experiment, lungs were lavaged twice with saline (2.5 ml per lavage). Lavage samples were centrifuged at $1,500 \mathrm{~g}$ at room temperature for $10 \mathrm{~min}$. The PCBAL was determined with a spectrophotometer by measuring the change in absorbance at $630 \mathrm{~nm}$ after the addition of bromocresol green $[6,20]$.

\section{Neutrophil elastase, myeloperoxidase, malondialdehyde and phospholipase $A_{2}$ activity}

The neutrophil elastase (NE) in lung perfusate was determined with a synthetic substrate, N-methoxysuccinyl-AlaAla-Pro-Val-p-nitroanilide as described previously [6,11]. In brief, samples were incubated in $0.1 \mathrm{M}$ Tris- $\mathrm{HCl}$ buffer (pH 8.0) containing $0.5 \mathrm{M} \mathrm{NaCl}$ and $1 \mathrm{mM}$ substrate at $37^{\circ} \mathrm{C}$ for $24 \mathrm{hr}$. After incubation, p-nitroanilide release was measured spectrophotometrically at $450 \mathrm{nM}$ and was considered NE activity.

To measure the myeloperoxidase (MPO) activity in lung perfusate, the samples were mixed with $2 \mathrm{ml}$ of potassium phosphate buffer $(50 \mathrm{mM}, \mathrm{pH}$ 6.0) containing $0.5 \%$ cetyltrimethylammonium bromide and were centrifuged at 2,500 g for $10 \mathrm{~min}$ at $4{ }^{\circ} \mathrm{C}$. The supernatant was diluted with dilution buffer, then mixed with an assay buffer composed of $0.00107 \% \mathrm{H}_{2} \mathrm{O}_{2}$ in potassium phosphate buffer and o-dianisidine. The reaction mixture was incubated at room temperature. The change in absorbance at $450 \mathrm{~nm}$ over $1 \mathrm{~min}$ was detected spectrophotometrically. The MPO activity was expressed as units per $\mathrm{ml}$ of lung perfusate using the absorbance of MPO standard (Elastine Products, Detroit, MC., USA). The procedures were basically followed those by Kinoshita et al. [8]. 
Malondialdehyde (MDA) was measured by thiobarbituric acid reaction. The principle of the method depends on the development of pink color produced by the interaction of barbituric acid with MDA as a result of lipid peroxidation. Tetraetoxypropane was used as standard $[21,22]$.

Plasma concentrations of phospholipase $\mathrm{A}_{2}\left(\mathrm{PLA}_{2}\right)$ were measured on a spectrofluorimeter using a method described by Kitsiouli et al. [23]. In brief, the standard incubation mixture contained $10 \mathrm{mmol} / \mathrm{l}$ tris/ $\mathrm{HCl}$ buffer (pH 7.4) with $2 \mathrm{mmol} / \mathrm{l} \mathrm{Ca} 2^{+}$and $5 \mu \mathrm{mil} / \mathrm{l} \mathrm{C}_{12}-\mathrm{NBD}-\mathrm{PC}$ \{1-palmitoyl-2-[12-[(7-nitro-2-1,3-benzoxadiazol-4-yl) amino]hexanoyl]-sn-glycero-3-phosphocholine 3 as the substrate. The absorbance of reaction mixture was measured with excitation and emission wavelengths at 475 and $535 \mathrm{~nm}$, respectively.

\section{Nitrate/nitrite, methyl guanidine, tumor necrosis factor $_{\alpha}$ and interleukin- $\mathbf{1}_{\beta}$}

Samples $(0.5 \mathrm{ml})$ were taken from the lung perfusate $1 \mathrm{hr}$ before and at various time points after drug administration. The samples were centrifuged at 3,000 g for $10 \mathrm{~min}$. The supernatant was used for determination of nitrate/ nitrite with high-performance chromatography [20,24]. The formation of methyl guanidine (MG) has been identified as an index of hydroxyl radical production [6,25]. It was determined with its fluorescence spectrum (Jasco 821FP, Spectroscopic Co., Tokyo, Japan). The emission maximum was set at $500 \mathrm{~nm}$ and the excitation maximum at $398 \mathrm{~nm}$. The assay was calibrated with authentic MG (Sigma M0377). Tumor necrosis factor ${ }_{\alpha}\left(\mathrm{TNF}_{\alpha}\right)$ and interleukin-1 $1_{\beta}\left(\mathrm{IL}-1_{\beta}\right)$ were measured with antibody enzymelinked immunosorbent assays (ELISAs) with a commercial antibody pair, recombinant standards, and a biotin-streptavdin-peroxidase detection system (Endogen, Rockford, IL, USA). All agents, samples, and working standards were prepared at room temperature according to the manufacturer's directions. The optical density was measured at 450/540 $\mathrm{nm}$ wavelengths by automated ELISA readers.

\section{Lung pathology}

Lung tissue was fixed in $10 \%$ formaldehyde for $24 \mathrm{hr}$ and then rinsed with tap water to remove formaldehyde. For light microscopic examination, lung tissue was dehydrated with graded alcohol and then embedded in paraffin at $60^{\circ} \mathrm{C}$. A series of microsections $(5 \mu \mathrm{m})$ was stained with hematoxylin and eosin. For quantification of lung injury score, we employed a modified grading method reported previously [6,24,25]. Various degree of lung injury score (LIS) were assessed as follows: degree 0, 1, 2, and 3 for no, mild, moderate and severe edema, respectively. For neutrophil and other cell infiltration, the scoring was similar to the evaluation of edema formation, degree 0-3 for no, mild, moderate and severe cellular infiltration. The histopathological assessment was performed in a blind fashion by several laboratory assistants. Each one gave a score for edema and cell infiltration from 0 to 3 . The individual scores for edema and cell infiltration were added together to obtain a final score, ranging from 0-6.

\section{Detection of iNOS and eNOS mRNA in lung tissue}

Reverse-transcriptase polymerase chain reaction (RTPCR) was employed for a semiquantitative detection of iNOS and eNOS mRNA expression [26].

\section{Drugs}

Phorbol myristate acetate (PMA) (Sigma Chemical, St Louis, MO, USA) was dissolved in dimethyl sulfoxide (DMSO; Sigma). Sivelestat (ONO-6818) was obtained from ONO company, Japan. It was dissolved in distilled water.

\section{Experimental protocol}

A total of 40 isolated lungs was randomly divided into 4 groups. The vehicle group received DMSO $100 \mu \mathrm{g} / \mathrm{g}$ lung weight. In the FE group, corn oil $0.6 \mathrm{ml}$ with distilled water $0.2 \mathrm{ml}$ was added into the lung perfusate $[5,6]$. The FE+PMA group received corn oil micelles with PMA (4 $\mu \mathrm{g} / \mathrm{g}$ lung weight) $[27,28]$. Sivelestat $(10 \mu \mathrm{g} / \mathrm{g}$ lung weight) was coadministered with corn oil micelles in $\mathrm{FE}+\mathrm{SVT}$ group. The dose was based on the dosage range from previous studies $[29,30]$. The isolated lungs were observed for $3 \mathrm{hr}$.

\section{Statistical analysis}

All data were expressed as mean \pm SEM. Comparisons within and among groups as well as data measurements over time in the same group were made using one-way analysis of variance with repeated measures, followed a post hoc comparison with Newman-Keuls test. Differences were considered to be statistically significant at $p<0.05$.

\section{Results}

\section{LW/BW ratio, LWG, exhaled NO and PCBAL}

In the isolated perfused lungs, FE caused ALI as evidenced by the increases in LW/BW ratio, LWG, exhaled $\mathrm{NO}$, and PCBAL (Figure 1). PAP and $\mathrm{K}_{\mathrm{fc}}$ were markedly elevated (Figure 2). Histopathological examination revealed severe lung edema and hemorrhage with inflammatory cell infiltration (Figure 3 ). The LIS was greatly larger than the vehicle control (Table 1). Biochemical determinations demonstrated significant increases in NE, MPO, MDA, PLA 2 , nitrate/nitrite, methyl guanidine, $\mathrm{TNF}_{\alpha}$, and IL- $1_{\beta}$ (Figures 4 and 5 ). The time course demonstrated that NE reached it's peak at $1 \mathrm{hr}$ after FE, $\mathrm{MPO}$ at $2 \mathrm{hr}, \mathrm{MDA}$ at $1.5 \mathrm{hr}$ and $\mathrm{PLA}_{2}$ at $2 \mathrm{hr}$ (Figure 4). 


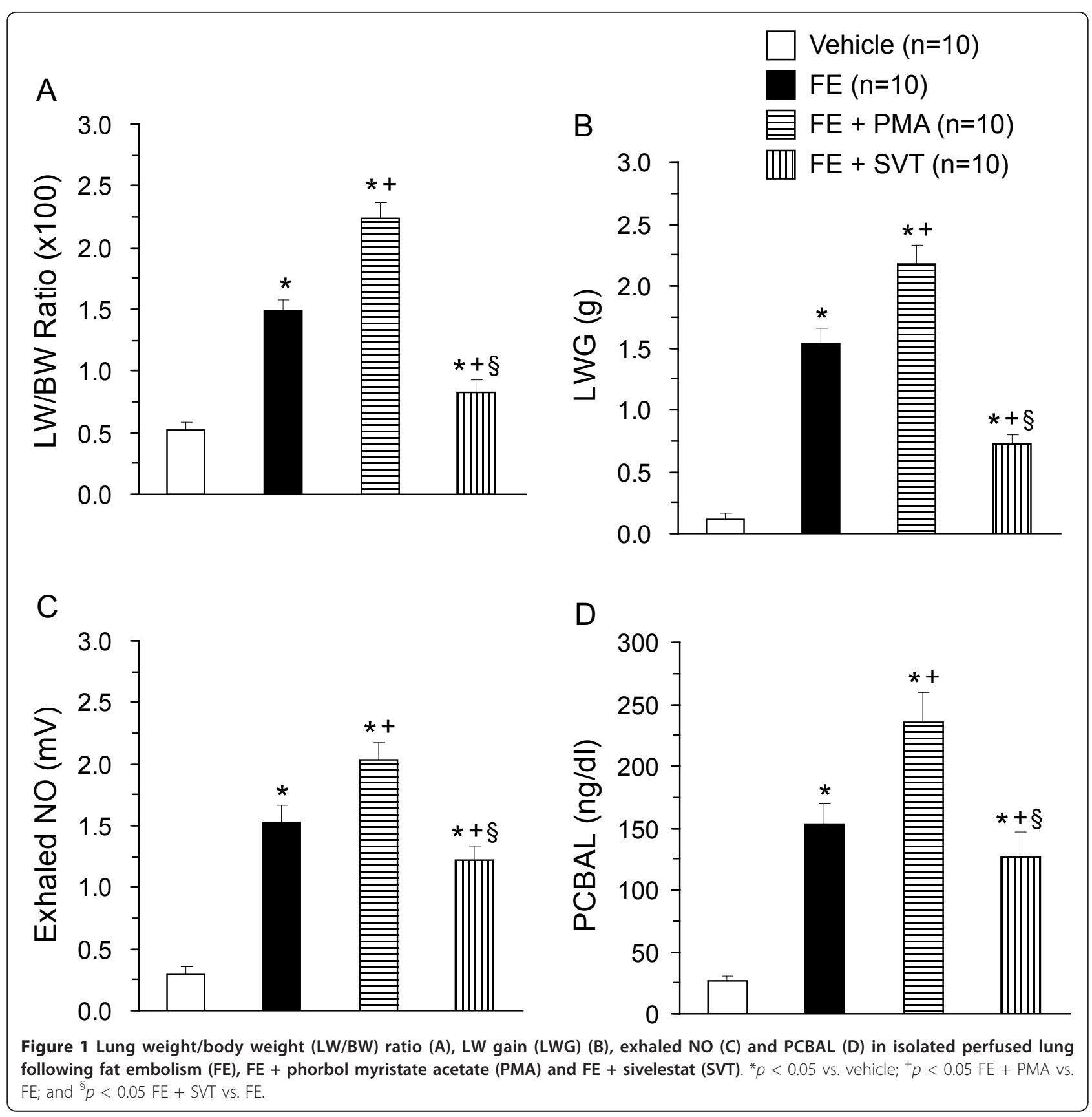

Nitrate/nitrite peaked at $1.5 \mathrm{hr}$, methyl guanidine at $1.5 \mathrm{hr}$, tumor necrosis factor ${ }_{\alpha}$ and interleukin- $1_{\beta}$ at $2 \mathrm{hr}$ (Figure 5). FE upregulated the iNOS expression significantly, while the eNOS was modestly increased (Figures 6A and 6B).

Addition of PMA significantly exacerbated the ALI induced by FE. The values of LW/BW, LWG, exhaled $\mathrm{NO}$, and PCBAL were elevated over those in the FE group (Figure 1). PMA also aggravated the FE-induced increases in pulmonary hypertension and capillary permeability (Figure 2). The lung pathology and LIS following FE were largely exacerbated by PMA (Figure 3 and
Table 1). In addition, PMA aggravated the FE-induced increases in NE, MPO, MDA and PLA 2 activities (Figure 4). It also increased the nitrate/nitrite, methyl guanidine, $\mathrm{TNF}_{\alpha}$ and IL- $1_{\beta}$ levels (Figure 5). This agent enhanced the iNOS expression significantly, while it elevated the eNOS mRNA modestly (Figure 6).

Administration of SVT significantly attenuated the FEinduced changes in lung parameters including LW/BW, LWG, exhaled NO, and PCBAL (Figure 1). It also reduced the PAP level and microvascular permeability (Figure 2). This neutrophil inhibitor abrogated the 


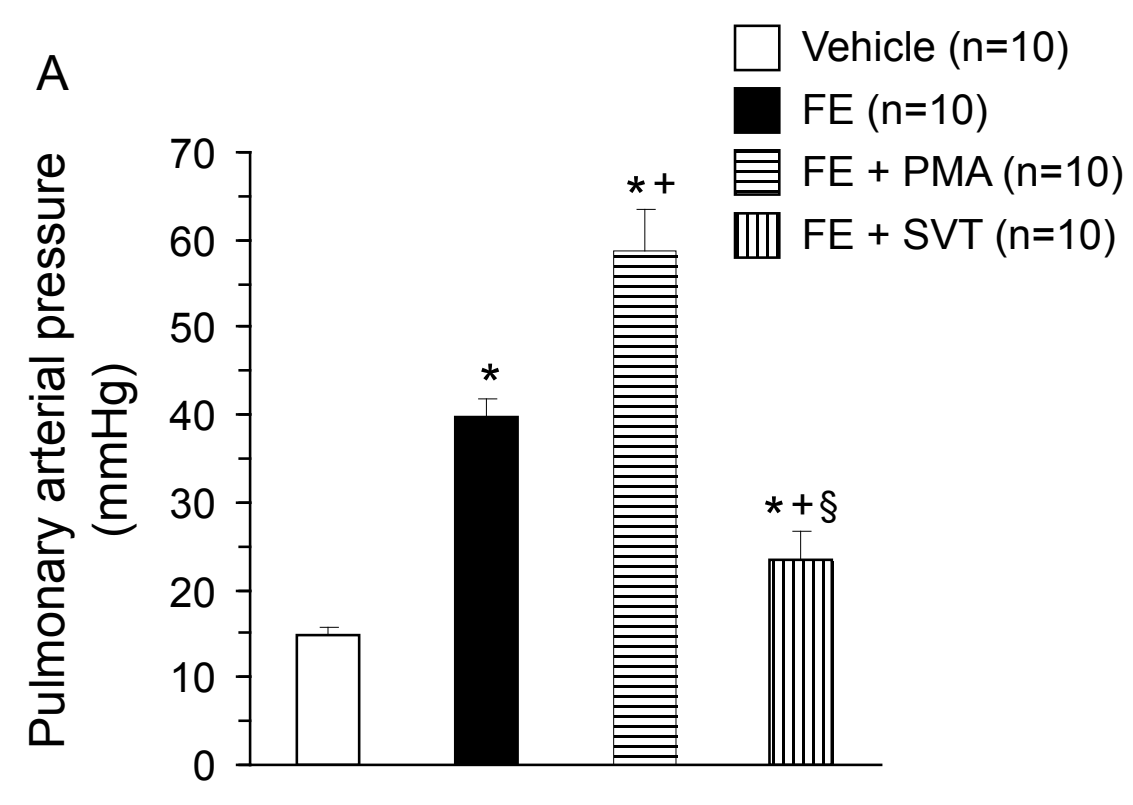

B

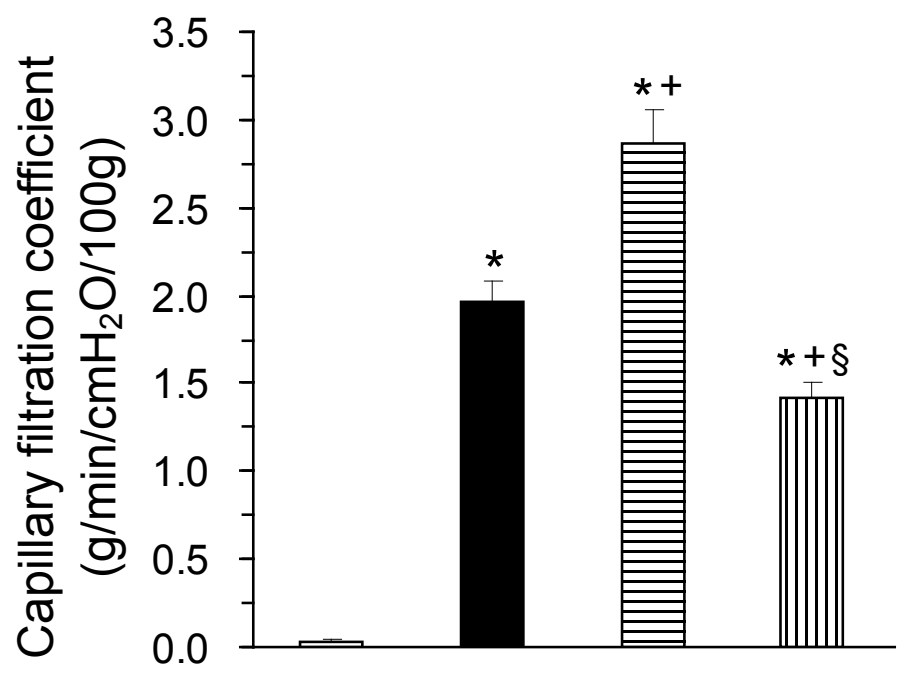

Figure 2 Increases in pulmonary arterial pressure (A) and microvascular permeability (B) in isolated lung caused by FE. Cotreatment with PMA enhanced the pulmonary hypertension and capillary filtration, while FE with SVT attenuated the changes in pulmonary arterial pressure and capillary permeability. ${ }^{*} p<0.05$ vs. vehicle; ${ }^{+} p<0.05 \mathrm{FE}+\mathrm{PMA}$ vs. FE; and ${ }^{\S} p<0.05 \mathrm{FE}+\mathrm{SVT}$ vs. FE.

histopathological lesions of the lung and snignificantly reduced the LIS (Figure 3 and Table 1). The NE, MPO, MDA, $\mathrm{PLA}_{2}$, nitrate/nitrite, methyl guanidine, $\mathrm{TNF}_{\alpha}$ and IL- $1_{\beta}$ in lung perfusate were decreased by SVT (Figures 4 and 5). The NE inhibitor attenuated the FE-induced upregulation of iNOS significantly, whereas the eNOS expression modestly (Figure 6).

\section{Discussion}

In the present study, we observed that fat embolism caused severe lung injury as evidenced by the changes in lung weight, exhaled NO, protein concentration in bronchoalveolar lavage fluid. In isolated lungs, FE caused severe lung injury associated with increases in pulmonary arterial pressure and microvascular permeability. The insult also resulted in increases in neutrophil elastase, myeloperoxidase, malondialdehyde and phospholipase $A_{2}$ activities. In addition, FE elevated the nitrate/nitrite, methyl guanidine, tumor necrosis factor ${ }_{\alpha}$ and interleukin- $1_{\beta}$ in lung perfusate. These pulmonary and biochemical changed were enhanced by coadministration with PMA, while mitigated by SVT. 

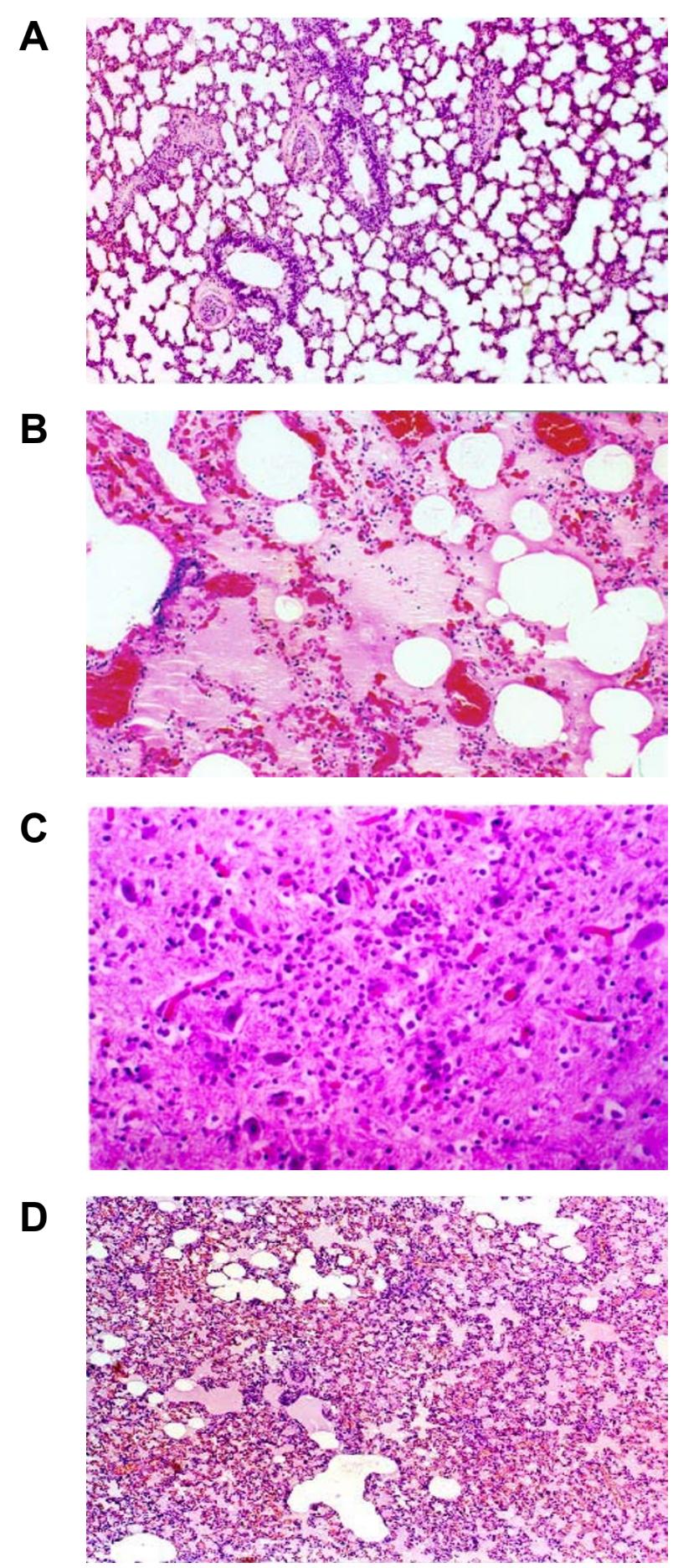

Figure 3 Histopathology of the lung tissue in vehicle (A), FE (B), FE + PMA (C) and FE + SVT (D) groups. FE caused severe alveolar edema and hemorrhage with inflammatory cell infiltration. Coadministration with PMA enhanced, but SVT diminished the lung pathology.

The pathogenesis of FE syndrome associated acute respiratory distress syndrome are complicated. Two clinical investigations from our laboratory have suggested that $\mathrm{NO}$, phospholipase $\mathrm{A}_{2}$, free radicals and proinflammatory cytokines are involved in the chemical phase. The major source of $\mathrm{NO}$ is the alveolar macrophages $[1,3]$. Animal studies have revealed that $\mathrm{N}$-acetylcysteine and $\mathrm{NO}$ inhibitors are able to abrogate the FE-induced changes $[6,7]$. 
Table 1 The lung injury score

\begin{tabular}{ll}
\hline Vehicle & $0.06 \pm 0.02$ \\
FE & $2.86 \pm 0.12^{*}$ \\
FE + PMA & $4.93 \pm 0.22^{*+}$ \\
FE + SVT & $1.42 \pm 0.08^{*+\S}$ \\
\hline
\end{tabular}

Values are mean \pm SEM ( $\mathrm{n}=10$ for each group). ${ }^{*} p<0.05$ vs. Vehicle; ${ }^{+} p<$ $0.05 \mathrm{FE}+\mathrm{PMA}$ and FE + SVT groups vs. FE group; ${ }^{\S} p<0.05 \mathrm{FE}+\mathrm{SVT}$ vs. FE + PMA. FE, fat embolism; PMA, phorbol myristate acetate; SVT, sivelestat.

The present study further provides evidence to indicate that NE inhibitor, sivelestat may be a therapeutic consideration for patients with FE syndrome.

Our observation of the time course of changes in mediators and proinflammatory cytokines may be valuable in the identification of the pathological subsequence of fat embolism. Neutrophil elastase may be released at the early chemical phase, followed by malondialdehyde, NO and hydroxyl radical $(1.5 \mathrm{hr})$, then myeloperoxidase, phospholipase $A_{2}$, tumor necrosis factor ${ }_{\alpha}$ and interleukin $-1_{\beta}(2 \mathrm{hr})$.
To verify this contention and identify the consequence of inflammatory cascade following FE, further studies using transgenic animals and specific inhibitors are required.

The physical phase of fat embolism syndrome caused pulmonary hypertension and increased capillary permeability because of pulmonary microvascular obstruction $[4,6]$. We indeed observed two phases of increases in PAP and LWG, an initial sharp upstroke followed by a slow rise (not shown). The changes may reflect the early physical phase and the late chemical phase of pulmonary hypertension and increased $\mathrm{K}_{\mathrm{fc}}$.

The increases in exhaled NO and nitrate/nitrite in lung perfusate, and NOS expression suggested that NO production was involved in the FE-induced ALI and associated changes. Our laboratory has reported that NO via the iNOS up-regulation contributed to the development of ARDS in children with enterovirus infection, patients with leptospirosis and scrub tybus and animal with hypercalcemia and endotoxemia [31-34]. In a murine model of

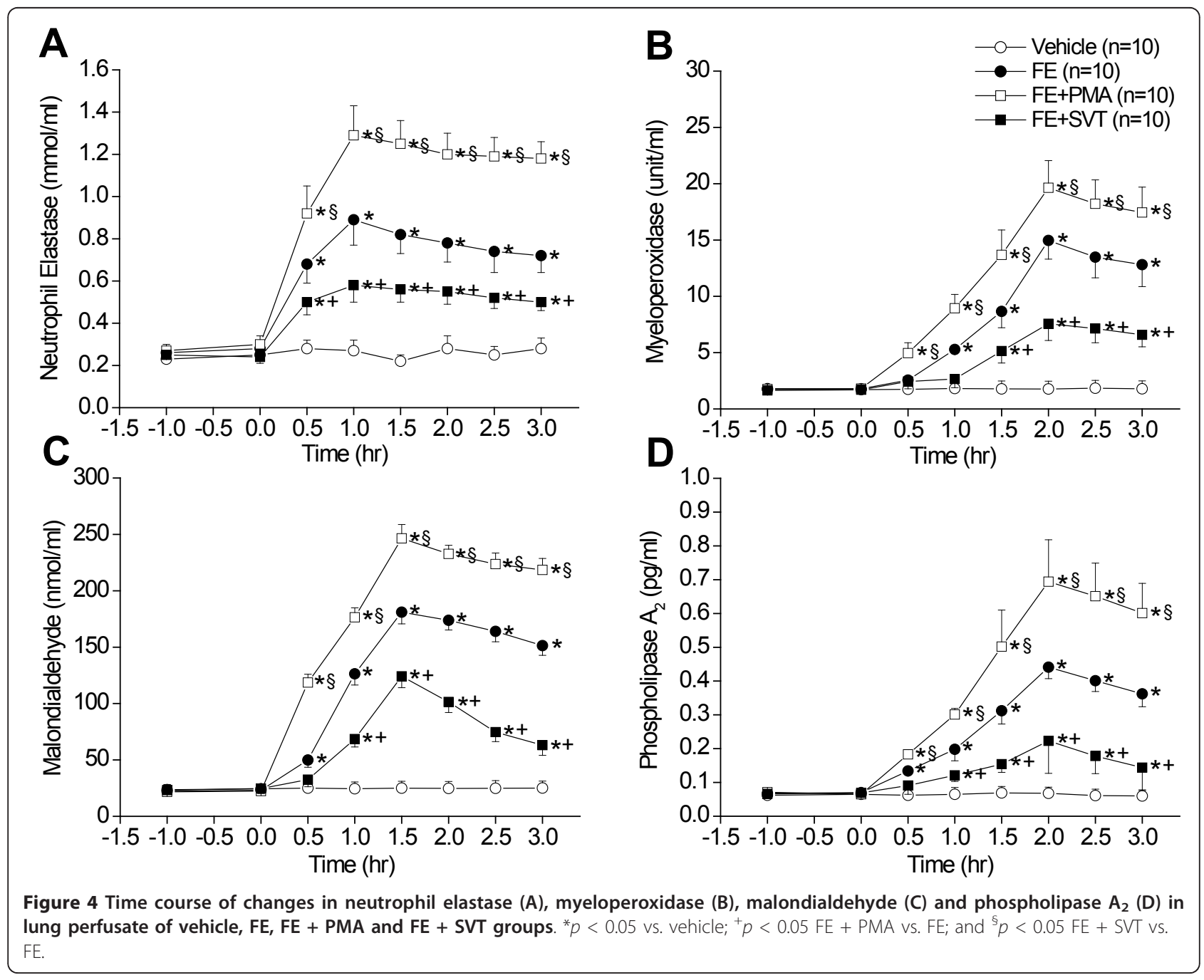




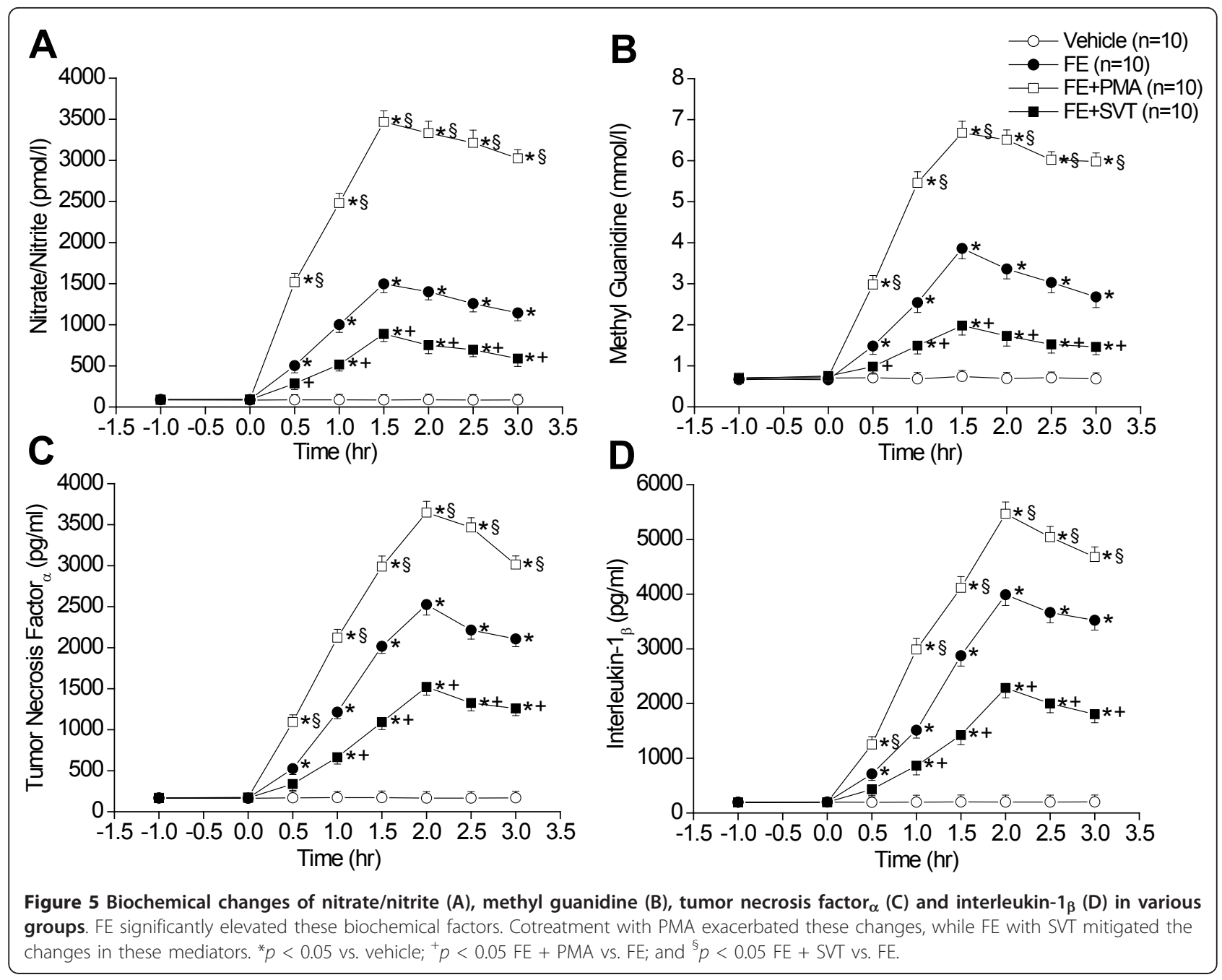

sepsis, Razava et al. proposed up-regulation of iNOS in the lung caused neutrophil infiltration. The recruitment iNOS-positive neutrophil further aggravated the oxidative stress in the lung [35]. We have also reported that pretreatment of iNOS isoform inhibitors attenuated the inflammatory responses to endotoxin, fat embolism and PMA. On the other hand, NO donors enhanced the pathological and biochemical changes $[7,27,36]$. Accordingly, NO production through the iNOS system plays a detrimental role in endotoxemia, infections and fat embolism as well. The participation of eNOS in the FE-induced changes appears to be of less importance.

Neutrophil activation and recruitment leading to release of neutrophil elastase, myeloperoxidase and malondialdehyde and other mediators have been proposed to be a important step leading to an inflammatory cascade. Pulmonary hypertension and increased microvascular permeability ensued. These alterations finally resulted in damage of the alveolar capillary barriers, thus created severe lung injury $[8,9,11]$. In the present study, the neutrophil-derived mediators were significantly elevated following fat embolism. The facilitatory effects of phorbol myristate acetate, while the inhibitory effects of sivelestat on the pathophysiological, biochemical and molecular changes induced by fat embolism further support the importance of neutrophil activation and release of neutrophil-derived mediators on the acute lung injury induced by fat embolism. In addition, phospholipase $A_{2}$ was increased in bronchoalveolar fluid and plasma of patients associated with fat embolism syndrome $[2,3]$. The ultimate role of phospholipase $\mathrm{A}_{2}$ in the FE-induced acute lung injury requires further investigation.

\section{Conclusions}

Fat embolism caused acute lung injury, pulmonary hypertension, increased microvascular permeability and lung pathology. The challenge in isolated and perfused rat's lungs also significantly elevated the neutrophil elastatase, myeloperoxidase, malondialdehyde and phopholipase $\mathrm{A}_{2}$ in lung perfusate. In addition, fat embolism caused 


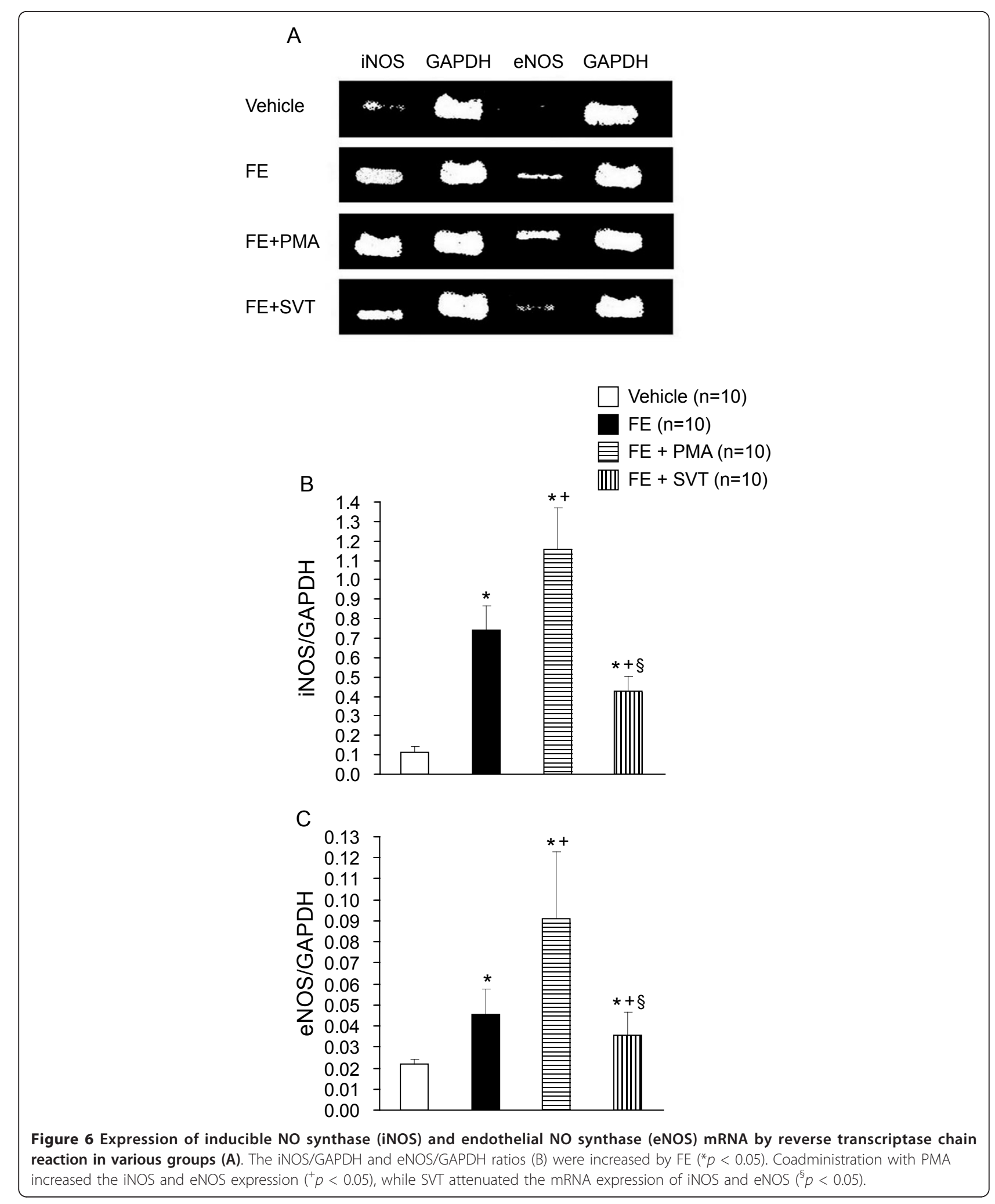


release of nitrate/nitrite, hydroxyl radical, tumor necrosis factor $r_{\alpha}$ and interleukin- $1_{\beta}$ from the lung. The time course revealed that neutrophil elastase was produced at the early chemical phase followed by other mediators. NO production through the iNOS exerts a detrimental effect on the lungs. Cotreatment with phorbol myristate acetate (a neutrophil elastase activator) exacerbated, while sivelestat (a neutrophil elastase inhibitor) attenuated the FE-induced changes. Neutrophil activation and neutrophil-derived mediators are involved in the FE syndrome associated with acute lung injury. Sivelestat may be recommended for clinical application in subjects with fat embolism syndrome.

\section{Acknowledgements}

This study was supported in part by a grant from the National Science Council (NSC-99-2320-B-320-010-MY3). We appreciate the technique and editing assistance from Ms. A. Huang.

\section{Author details}

'Department of Physical Education and Kinesiology, National Dong Hwa University, Hualien, Taiwan. ${ }^{2}$ Department of Anatomy, Tzu Chi University, Hualien, Taiwan. ${ }^{3}$ School of Respiratory Therapy, Fu Jen Catholic University and Taipei Medical University, New Taipei City, Taiwan. ${ }^{4}$ Department of Internal Medicine, Shin Kong Wu Ho-Su Memorial Hospital, New Taipei City, Taiwan. ${ }^{5}$ Institute of Physiological and Anatomical Medicine, Tzu Chi University, Hualien, Taiwan.

\section{Authors' contributions}

CCL and PHL performed the animal experiments. PHL also carried out pathological examination of the lung, and his assistants assessed the lung injury score. SJK was in charge of the data collection and analysis. HIC was the principal investigator to obtain the grant. He contributed to the study design, coordination, manuscript writing and correspondence. All authors read and approved the final manuscript.

\section{Competing interests}

The authors declare that they have no competing interests.

Received: 4 November 2011 Accepted: 5 January 2012

Published: 5 January 2012

\section{References}

1. Hsu YH, Kao SJ, Lee RP, Chen HI: Acute pulmonary oedema: rare causes and possible mechanisms. Clin Sci (Lond) 2003, 104:259-264.

2. Karagiorga G, Nakos G, Galiatsou E, Lekka ME: Biochemical parameters of bronchoalveolar lavage fluid in fat embolism. Intensive Care Med 2006, 32:116-123.

3. Kao SJ, Yeh DY, Chen HI: Clinical and pathological features of fat embolism with acute respiratory distress syndrome. Clin Sci (Lond) 2007, 113:279-285.

4. White T, Petrisor BA, Bhandari M: Prevention of fat embolism syndrome. Injury 2006, 37:S59-S67.

5. Liu DD, Hsieh NK, Chen HI: Histopathological and biochemical changes following fat embolism with administration of corn oil micelles: a new animal model for fat embolism syndrome. J Bone Joint Surg Br 2008, 90:1517-1521.

6. Liu DD, Kao SJ, Chen HI: N-acetylcysteine attenuates acute lung injury induced by fat embolism. Crit Care Med 2008, 36:565-571.

7. Kao SJ, Chen HI: Nitric oxide mediates acute lung injury caused by fat embolism in isolated rat's lungs. J Trauma 2008, 64:462-469.

8. Kinoshita M, Ono S, Mochizuki H: Neutrophils mediate acute lung injury in rabbits: role of neutrophil elastase. Eur Surg Res 2003, 32:337-346.

9. Abraham E: Neutrophils and acute lung injury. Crit Care Med 2003, 31 S195-S199.
10. Lee WL, Downey GP: Leukocyte elastase: physiological functions and role in acute lung injury. Am J Respir Crit Care Med 2001, 164:896-904.

11. Kuraki T, Ishibashi M, Takayama M, Shiraishi M, Yoshida M: A novel oral neutrophil elastase inhibitor (ONO-6818) inhibits human neutrophil elastase-induced emphysema in rats. Am J Respir Crit Care Med 2002, 166:496-500.

12. Murakami K, Okajima K, Uchiba M: The prevention of lipopolysaccharideinduced pulmonary vascular injury by pretreatment with cepharanthine in rats. Am J Respir Crit Care Med 2000, 161:57-63.

13. Murakami K, Cox RA, Hawkins HK, Schmalstieg FC, McGuire RW, Jodoin JM, Traber LD, Traber DL: Cepharanthin, an alkaloid from Stephania cepharantha, inhibits increased pulmonary vascular permeability in an ovine model of sepsis. Shock 2003, 20:46-51.

14. Inoue Y, Seiyama A, Tanaka H, Ukai I, Akimau P, Nishino M, Shimazu T, Sugimoto H: Protective effects of a selective neutrophil elastase inhibitor (sivelestat) on lipopolysaccharide-induced acute dysfunction of the pulmonary microcirculation. Crit Care Med 2005, 33:1814-1822.

15. Hagiwara S, Iwasaka H, Togo K, Noguchi T: A neutrophil elastase inhibitor, sivelestat, reduces lung injury following endotoxin-induced shock in rats by inhibiting HMGB1. Inflammation 2008, 31:227-234.

16. Fujii M, Miyagi Y, Bessho R, Nitta T, Ochi M, Shimizu K: Effect of a neutrophil elastase inhibitor on acute lung injury after cardiopulmonary bypass. Interact Cardiovasc Thorac Surg 2010, 10:859-862.

17. Ando M, Murai T, Takahashi $Y:$ The effect of sivelestat sodium on postcardiopulmonary bypass acute lung injury in a neonatal piglet model. Interact Cardiovasc Thorac Surg 2008, 7:785-788.

18. Kao SJ, Wang D, Yeh DY, Hsu K, Hsu YH, Chen HI: Static inflation attenuates ischemia/reperfusion injury in an isolated rat lung in situ. Chest 2004, 126:552-558.

19. Stewart TE, Valenza F, Ribeiro SP, Wener AD, Volgyesi G, Mullen JB, Slutsky AS: Increased nitric oxide in exhaled gas as an early marker of lung inflammation in a model of sepsis. Am J Respir Crit Care Med 1995, 151:713-718.

20. Su CF, Liu DD, Kao SJ, Chen HI: Nicotinamide abrogates acute lung injury caused by ischaemia/reperfusion. Eur Respir J 2007, 30:199-204.

21. Bioshop ML, Janet LP: Free Radicals in Clinical Chemistry. Third edition. Philadelphia, Lippincott; 1996.

22. Chen HI, Hsieh NK, Kao SJ, Su CF: Protective effects of propofol on acute lung injury induced by oleic acid in conscious rats. Crit Care Med 2008, 36:1214-1221.

23. Kitsiouli El, Nakos G, Lekka ME: Differential determination of phospholipase A(2) and PAF-acetylhydrolase in biological fluids using fluorescent substrates. J Lipid Res 1999, 40:2346-2356.

24. Chen HI, Yeh DY, Liou HL, Kao SJ: Insulin attenuates endotoxin-induced acute lung injury in conscious rats. Crit Care Med 2006, 34:758-764.

25. Kao SJ, Liu DD, Su CF, Chen HI: Niacinamide abrogates the organ dysfunction and acute lung injury caused by endotoxin. J Cardiovasc Pharmacol 2007, 50:333-342.

26. Lin NT, Yang FL, Lee RP, Peng TC, Chen HI: Inducible nitric oxide synthase mediates cytokine release: the time course in conscious and septic rats. Life Sci 2006, 78:1038-1043.

27. Yang YL, Huang KL, Liou HL, Chen HI: The involvement of nitric oxide, nitric oxide synthase, neutrophil elastase, myeloperoxidase and proinflammatory cytokines in the acute lung injury caused by phorbol myristate acetate. J Biomed Sci 2008, 15:499-507.

28. Chu SJ, Chang DM, Wang D, Hsu K, Chiang CH: Protective effect of lipophilic antioxidants on phorbol-induced acute lung injury in rats. Crit Care Med 2001, 29:819-824.

29. Wang HG, Shibamoto T, Miyahara T, Haniu H, Tanaka S, Fujimoto K, Honda T, Kubo K, Koyama S: Effect of ONO-5046, a specific neutrophil elastase inhibitor, on the phorbol myristate acetate-induced injury in isolated dog lung. Exp Lung Res 1999, 25:55-67.

30. Miyazaki Y, Inoue T, Kyi M, Sawada M, Miyake S, Yoshizawa Y: Effects of a neutrophil elastase inhibitor (ONO-5046) on acute pulmonary injury induced by tumor necrosis factor alpha (TNFalpha) and activated neutrophils in isolated perfused rabbit lungs. Am J Respir Crit Care Med 1998, 157:89-94

31. Kao SJ, Yang FL, Hsu YH, Chen HI: Mechanism of fulminant pulmonary edema caused by enterovirus 71. Clin Infect Dis 2004, 38:1784-1788.

32. Chen HI, Kao SJ, Hsu YH: Pathophysiological mechanism of lung injury in patients with leptospirosis. Pathology 2007, 39:339-344. 
33. Chen HI, Yeh DY, Kao SJ: The detrimental role of inducible nitric oxide synthase in the pulmonary edema caused by hypercalcemia in conscious rats and isolated lungs. J Biomed Sci 2008, 15:227-238.

34. Hsu YH, Chen HI: Pulmonary pathology in patients associated with scrub typhus. Pathology 2008, 40:268-271.

35. Razavi HM, Wang le F, Weicker S, Rohan M, Law C, McCormack DG, Mehta S: Pulmonary neutrophil infiltration in murine sepsis: role of inducible nitric oxide synthase. Am J Respir Crit Care Med 2004, 170:227-233.

36. Su CF, Yang FL, Chen HI: Inhibition of inducible nitric oxide synthase attenuates acute endotoxin-induced lung injury in rats. Clin Exp Pharmacol Physiol 2007, 34:339-346.

doi:10.1186/1423-0127-19-3

Cite this article as: Lin et al:: Effects of phorbol myristate acetate and sivelestat on the lung injury caused by fat embolism in isolated lungs. Journal of Biomedical Science 2012 19:3.

\section{Submit your next manuscript to BioMed Central} and take full advantage of:

- Convenient online submission

- Thorough peer review

- No space constraints or color figure charges

- Immediate publication on acceptance

- Inclusion in PubMed, CAS, Scopus and Google Scholar

- Research which is freely available for redistribution

Submit your manuscript at www.biomedcentral.com/submit
C Biomed Central 